\title{
SLAM, a Mathematica interface for SUSY spectrum generators
}

\author{
Peter Marquard ${ }^{\mathrm{a}}$, Nikolai Zerf ${ }^{\mathrm{b}, *}$ \\ a Deutsches Elektronen Synchrotron DESY, Platanenallee 6, D15738 Zeuthen, Germany \\ ${ }^{\mathrm{b}}$ Department of Physics, University of Alberta, Edmonton AB T6G 2J1, Canada
}

\section{A R T I C L E I N F O}

\section{Article history:}

Received 19 September 2013

Received in revised form

27 November 2013

Accepted 3 December 2013

Available online 11 December 2013

\section{Keywords:}

Mathematica package

Supersymmetry Les Houches Accord

MSSM

Spectrum

SQL data base

\begin{abstract}
A B S T R A C T
We present and publish a Mathematica package, which can be used to automatically obtain any numerical MSSM input parameter from SUSY spectrum generators, which follow the SLHA standard, like SPheno, SOFTSUSY, SuSeFLAV or Suspect. The package enables a very comfortable way of numerical evaluations within the MSSM using Mathematica. It implements easy to use predefined high scale and low scale scenarios like mSUGRA or $m_{h}^{\max }$ and if needed enables the user to directly specify the input required by the spectrum generators. In addition it supports an automatic saving and loading of SUSY spectra to and from a SQL data base, avoiding the rerun of a spectrum generator for a known spectrum.
\end{abstract}

\section{Program summary}

Program title: SLAM

Catalogue identifier: AERX_v1_0

Program summary URL: http://cpc.cs.qub.ac.uk/summaries/AERX_v1_0.html

Program obtainable from: CPC Program Library, Queen's University, Belfast, N. Ireland

Licensing provisions: Standard CPC licence, http://cpc.cs.qub.ac.uk/licence/licence.html

No. of lines in distributed program, including test data, etc.: 4387

No. of bytes in distributed program, including test data, etc.: 37748

Distribution format: tar.gz

Programming language: Mathematica.

Computer: Any computer where Mathematica version 6 or higher is running providing bash and sed.

Operating system: Linux.

Classification: 11.1.

External routines: A SUSY spectrum generator such as SPheno, SOFTSUSY, SuSeFLAV or SUSPECT

Nature of problem:

Interfacing published spectrum generators for automated creation, saving and loading of SUSY particle spectra.

Solution method:

SLAM automatically writes/reads SLHA spectrum generator input/output and is able to save/load generated data in/from a data base.

Restrictions:

No general restrictions, specific restrictions are given in the manuscript.

Running time:

A single spectrum calculation takes much less than one second on a modern PC.

(c) 2013 Elsevier B.V. All rights reserved.

\footnotetext{
th This paper and its associated computer program are available via the Computer Physics Communication homepage on ScienceDirect (http://www.sciencedirect.com/ science/journal/00104655).

* Corresponding author. Tel.: +1 7802481098.

E-mail addresses: peter.marquard@desy.de (P. Marquard), zerf@ualberta.ca (N. Zerf).
} 


\section{Introduction}

Although there has been no experimental evidence for the realization of Supersymmetry (SUSY) yet, the Minimal Supersymmetric Standard Model (MSSM) became very popular among particle physicists. Many calculations of elementary particle processes include the effects of SUSY particles. This applies to cosmological predictions like relic density of dark matter from early universe, predictions of cross sections for the direct production of SUSY particles at colliders like the LHC and ILC, the calculation of radiative corrections due to the presence of SUSY particles for Standard Model (SM) processes, for example in flavour physics, and many more.

Compared to the SM a supersymmetric model is more predictive because supersymmetry imposes many relations between different parameters. However, due to the fact that we do not live in a supersymmetric world SUSY must be softly broken in a realistic model describing our world. Since the breaking mechanism is still unknown many new unknown parameters arise in the broken model.

Once one makes an assumption for those unknown parameters or assumes a certain breaking mechanism of SUSY, many parameters can be determined directly from the knowledge of the measured SM parameters. For example, the Higgs mass is an independent parameter in the SM. In the MSSM this is no longer true, because after assuming a certain SUSY breaking model it can be calculated from the knowledge of a few parameters. On the one hand this is a nice feature, but on the other hand it leads to the problem of a consistent determination of all relevant parameters like masses, mixing angles, and couplings in such a model. A collection of such a set of parameters will be called spectrum in the following.

Fortunately this problem has already been solved and spectra can be calculated automatically using spectrum generators like SPheno [1], SOFTSUSY [2], SuSeFLAV [3] or Suspect [4].

In order to manage the identification of particle parameters and couplings as well as option settings for programs in a common way, the Supersymmetry Les Houches Accord (SLHA) has been proposed $[5,6]$ and is used by many programs including the mentioned ones. ${ }^{1}$ Within the SLHA the generation of a consistent spectrum can be specified by providing a single input file (LesHouches.in). Providing this file leads, after a run of the generator, to an output file (LesHouches . out) containing the complete spectrum in the SLHA notation.

If one is interested in only a few parameters of a single spectrum one can easily extract them via copy and paste by hand. However, this procedure is certainly not feasible if one needs to extract many parameters of more than one spectrum for further numerical evaluation. In fact this is the situation one is facing immediately when trying to create a plot in dependence of high or low scale scenario parameters like $\tan \beta$. That means in order to be able to use the output of spectrum generators or any other program using SLHA, one needs an interface providing an automatic extraction of the relevant parameters from the output file LesHouches . out.

There already exist public interfaces written for C++ [8-11], FORTRAN [11-13], and Python [14].

Due to its vast amount of implemented functions and with increasing computer power and increasing computation speed, Mathematica became an attractive alternative for numerical evaluation of analytic expressions obtained in SUSY models. However, to the authors' knowledge there is up to now no public implementation of an interface to spectrum generators, automatically writing and reading SLHA files with the goal to provide simple data sets containing all needed parameters in Mathematica for general ${ }^{2}$ purpose.

This task is accomplished by SLAM (Supersymmetry Les Houches Accord with Mathematica) which will be presented in detail throughout this publication. A preliminary version of this package with name LHSQLDB was already used in Refs. [16] and [17].

This paper is organized as follows: for the impatient reader we present in Section 2 typical usage examples which should give a compact overview of what SLAM is capable to do. In Section 3 we give detailed information about how to install and configure the package properly before we give a full usage instruction in Section 4 . Section 5 is dedicated to the internal structure of SLAM and may help interested users to go beyond the "black box" model of this package.

\section{Teaser examples}

To demonstrate, how the package should be used, we present some of the implemented scenarios in this section. The full list of predefined scenarios can be found in Table 2 .

\subsection{Predefined $m_{h}^{\max }$-scenario}

Once a spectrum generator is installed on the system and SLAM is configured properly it is very easy to obtain a SLHA spectrum within Mathematica. Install the package into the Mathematica kernel by loading the main file:

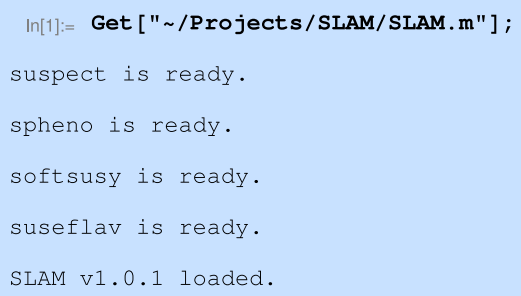

Afterwards, one can generate spectra, e.g. in the predefined $m_{h}^{\max }$-scenario [18] by using the central function 0btainLesHouchesSpectrum:

\footnotetext{
1 A list of programs using SLHA can be found in Ref. [7].

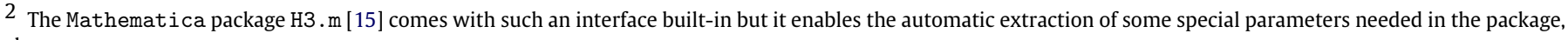
only.
} 


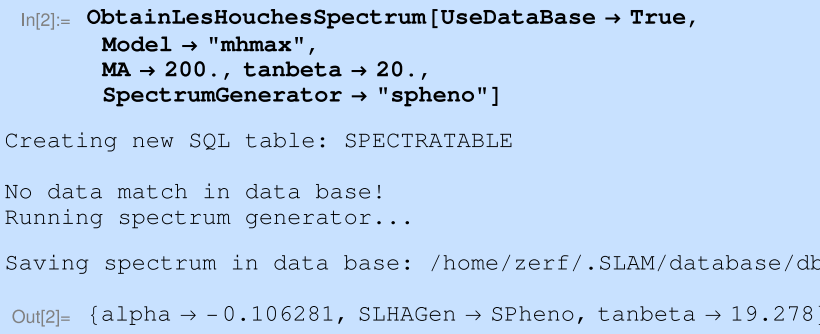

With the displayed command we have requested a $m_{h}^{\max }$-spectrum using the spectrum generator SPheno. Such a spectrum depends on $M_{A}$ and $\tan \beta$. Because we did not switch off the usage of a currently empty data base, SLAM creates a new table in it. Since there is no matching spectrum in the data base the spectrum generator SPheno is run with the given parameters and the spectrum stored in the data base.

Finally it returns a part of the spectrum in a list of replacement rules. Since no parameters have been requested explicitly, SLAM just returned a list of selected parameters, which are defined in a default request list.

Assuming we are only interested in the mass of the lightest Higgs boson $M_{h^{0}}$ within the same scenario we can use:

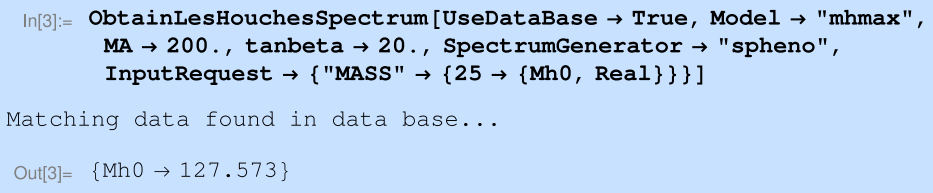

where we have used the SLHA identification for the on-shell Higgs mass, settled in the Block "MASS" with key entry 25. The choice of the symbol MhO is completely arbitrary, up to the restriction that it has to be an undefined symbol. This allows the user to use his own notation in Mathematica. The Real statement tells SLAM that it should treat the parameter during the internal processing as a real valued number. Note that SLAM did not rerun SPheno to obtain the Higgs mass, but just retrieved the value directly from the data base, where it has been stored during the previous call of the function ObtainLesHouchesSpectrum.

In order to see the default value of the option InputRequest one can use:

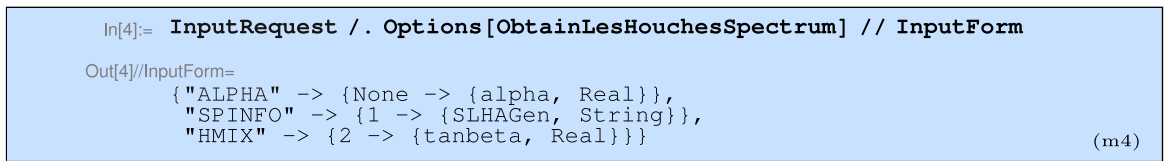

With the given output it is straightforward to customize the InputRequest option values for the user's needs.

Besides the $m_{h}^{\max }$ scenario the no-mixing, gluophobic and small $\alpha_{\text {eff }}$ scenarios, which were proposed together with the $m_{h}^{\max }$ scenario in Ref. [18], are already implemented and have the same input variables $M_{A}$ and $\tan \beta$. They are used when setting the Model option to "nomix", "smallalpha", or "gluophob".

\subsection{Predefined mSUGRA-scenario}

The mSUGRA-scenario exists as a predefined model and can be called as follows:

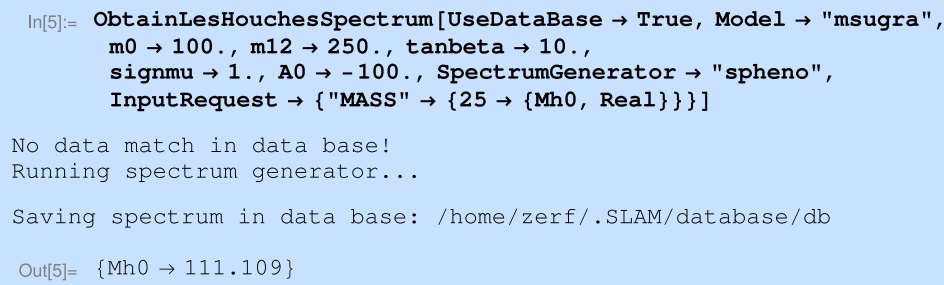

The shown values for the five input parameters represent their default values. One should be aware that those values are already excluded experimentally and their allowed values are much higher.

\subsection{Predefined p19MSSM plane I/II scenario}

Since more and more space of the MSUGRA scenario has been excluded at the LHC, there has been a proposal of many scenarios in Ref. [19] taking into account exclusions by this non-observation of SUSY particles. If there is no interest in a SUSY breaking mechanism motivated by some high scale physics, one can just define all the SUSY breaking parameters at a low scale. With certain assumptions one can reduce the number of these parameters to 19 . 
Two different planes have been defined in such a phenomenological 19 parameter MSSM [19]:

- p19MSSM plane I ("p19MSSM1") in dependence of $M_{1}(\mathrm{M} 1)$ and $M_{3}(\mathrm{M} 3)$.

- p19MSSM plane II ("p19MSSM2") depends on $M_{1}\left(\right.$ M1) and a common soft slepton mass breaking parameter $m_{\tilde{l}}$ (mslepton) of the first two generations.

Both scenarios are implemented in SLAM. We just show an example for the first one:

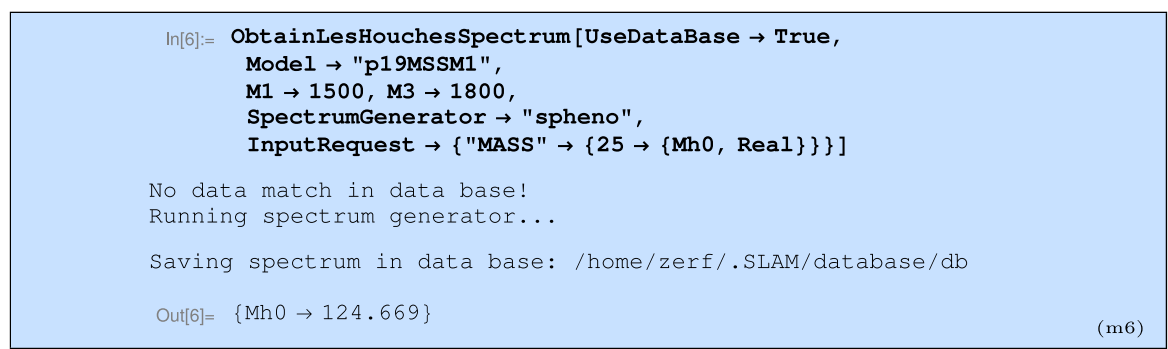

To provide a reasonable light Higgs mass, we show the SPheno result for the scenario point p19MSSM1.13. The very first points in the sequence (p19MSSM1.1, p19MSSM1.2, ...) seem to be already excluded if one assumes that the new particle discovered at the LHC is the lightest Higgs boson.

\subsection{User-defined scenarios}

On the one hand predefined scenarios are quite useful, because the user does not need to worry about all the required input parameters, on the other hand they are very restrictive. To remove this disadvantage, SLAM can be used to generate spectra for any user-defined scenario one can think of.

This is done by handing all relevant input parameters to SLAM directly via the option Model of the function ObtainLesHouchesSpectrum. In the example below we generate the same mSUGRA spectrum like two sections ago but this time using direct model input for the underlying scenario:

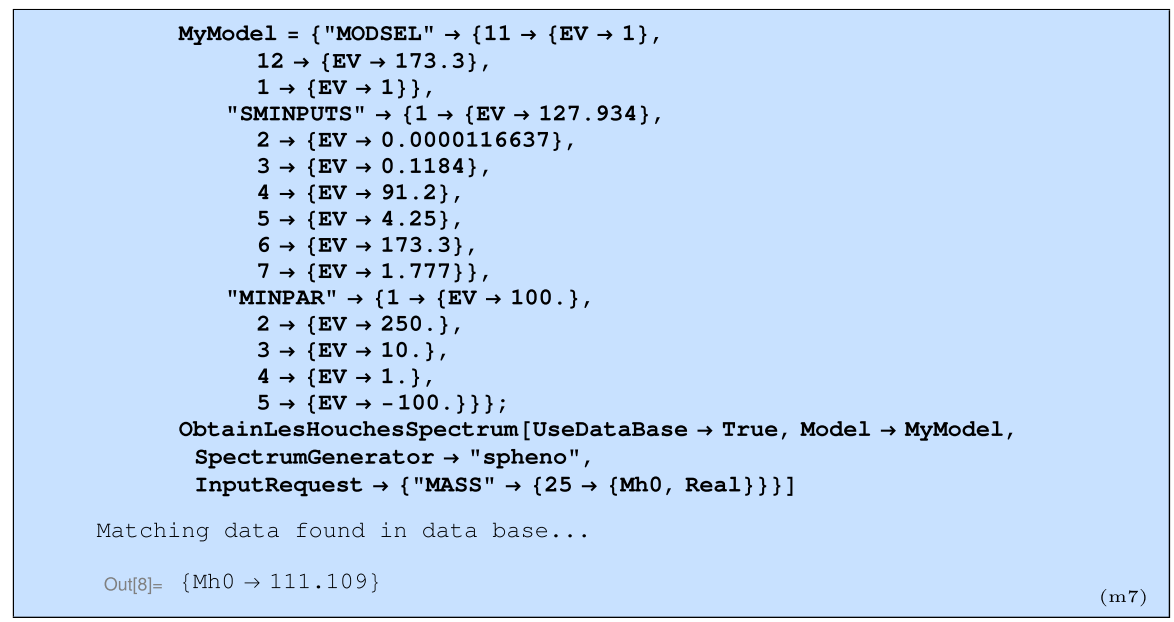

In the first line we defined our own model in variable MyModel. In this example, we use a minimal definition of the model, only giving the definition of needed entry values which are indicated by the EV symbols. In addition, it is possible to define comments for every entry, which can improve the readability of the SLHA files.

As can be seen from the output, the requested spectrum was already stored in the data base, which is the case since it corresponds to the predefined mSUGRA scenario used before.

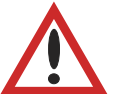
We have to add the warning that when using user-defined scenarios, the user has to make sure that the input provided is sufficient for the spectrum generators to run correctly. SLAM will not check the provided input for its consistency.

\section{Prerequisites \& installation}

\subsection{Prerequisites}

To be able to use SLAM one needs:

- Mathematica of version 6.0 or higher.

- A UNIX operating system providing bash and sed. 
And at least one of the spectrum generators listed in the following table.

\begin{tabular}{lcc}
\hline Generator & Weblink & Version \\
\hline SPheno & http://spheno.hepforge.org/ & 3.2 .0 \\
SOFTSUSY & http://softsusy.hepforge.org/ & 3.3 .4 \\
SuSeFLAV & https://suseflav.hepforge.org/ & 1.2 .0 \\
Suspect & http://www.coulomb.univ-montp2.fr & 2.4 .3 \\
\hline
\end{tabular}

They can be obtained from the stated websites. The versions indicated have been used for this publication, ${ }^{3}$ but SLAM is not restricted to them.

A running SQL server may be very convenient for quickly storing and loading spectrum data. But it is not mandatory, because Mathematica has a built-in data base server. ${ }^{4}$

\subsection{Installation}

Installing SLAM is quite simple:

- Download the package from [20].

- Unpack the tarball and put the two files:

- SLAM.m

- SLAM.config.m

in a common folder.

- Open SLAM . config.m with any text editor and adjust the given entries appropriately.

To clarify the last step, we give detailed information about every setting contained in SLAM.config.m, although the contained comments should be sufficient to adjust the settings without manual.

The configuration file SLAM. config.m is - like the extension suggests - obeying Mathematica syntax and any change in this file must not break it. In the following we describe the contained settings.

The first set of options has to be adjusted to reflect the user's setup

- ProgramPath ["spheno"]="/.../SPheno-3.2.0/bin/SPheno" ProgramPath ["softsusy"]="/.../softsusy-3.3.4/bin/softpoint.x"

ProgramPath ["suseflav"]="/.../SuSeFLAV-1.2/bin/suseflavslha"

ProgramPath ["suspect"]="/.../suspect2/suspect2"

define the path to the SPheno, SOFTSUSY, SuSeFLAV and Suspect executables on the file system as a string.

- SQLDataBaseTypeValue-> "hsqldb"

sets the data base type to the default data base server included in Mathematica. To connect to a dedicated SQL server this settings might change to the following:

SQLDataBaseTypeValue-> "MySQL (Connector/J)"

To be more specific this setting is used as an argument when connecting to the data base with the command:

OpenSQLConnection [JDBC [SQLDataBaseTypeValue, .... . . . .

One can use the Mathematica built-in connection tool

Needs ["DatabaseLink'"];

OpenSQLConnection []

to find/set the proper definition of the needed values here and below. More details can be found in the documentation centre of

Mathematica.

- SQLDataBaseOptionsValue->

\{"Name" ->"Spectrum", "Username" ->Environment ["USER"]\}

contains a list of options relevant for the data base.

When using a SQL server the list might look as follows:

\{"Username" ->Environment ["USER"], "Password" -> "xxx",

"Catalog" ->"..."

This list is used when connecting to the data base: OpenSQLConnection [JDBC [... ], SQLDataBaseOptionsValue]

- SQLDataBaseValue ->

(Environment ["HOME"] <>"/.SLAM/database/db")

gives the link to the data base as a string. When using the built-in Mathematica data base, this is the location on the system where Mathematica will store its data base files. In case a dedicated SQL server is used, this string contains its address including port information:

SQLDataBaseValue ->"sqlserver.someaddress.de:3306"

The option is used as a second argument during the connection to the data base:

OpenSQLConnection [JDBC [ . . , SQLDataBaseValue] ,....

\footnotetext{
3 using different versions may result in slightly different output

4 which however slows down when dealing with a large number $(>1000)$ of saved spectra
} 
Table 1

Default definition of numerical values used in SLAM in proper powers of $\mathrm{GeV}$. The circled numbers show the ID of the corresponding model, where the specific default value is used. See Table 2 for more details.

\begin{tabular}{|c|c|c|}
\hline Variable & $\begin{array}{l}\text { Symbol } \\
\text { Default value }\end{array}$ & Description \\
\hline invalphaValue & $\begin{array}{l}\alpha^{-1, \overline{\mathrm{MS}}}\left(m_{Z}\right) \\
1.279340000 \cdot 10^{2}\end{array}$ & Inverse fine structure constant \\
\hline GFValue & $\begin{array}{l}G_{F} \\
1.16637 \cdot 10^{-5}\end{array}$ & Fermi-constant \\
\hline asmzValue & $\begin{array}{l}\alpha_{s}^{(5), \overline{\mathrm{MS}}}\left(m_{Z}\right) \\
0.1184\end{array}$ & QCD fine structure constant \\
\hline MwValue & $\begin{array}{l}m_{W} \\
80.399\end{array}$ & $W$ boson mass \\
\hline MzValue & $\begin{array}{l}m_{Z} \\
91.200\end{array}$ & $Z$ boson mass \\
\hline MbMSbarValue & $\begin{array}{l}m_{b}^{\overline{\mathrm{MS}}}\left(m_{b}\right) \\
4.25\end{array}$ & $b$ quark mass \\
\hline MtOSValue & $\begin{array}{l}M_{t} \\
173.3\end{array}$ & $t$ quark pole mass \\
\hline MtauValue & $\begin{array}{l}m_{\tau} \\
1.777\end{array}$ & $\tau$ lepton pole mass \\
\hline moValue & $\begin{array}{l}m_{0} \\
1.0 \cdot 10^{2}\end{array}$ & Common scalar mass (1) (3) \\
\hline m12Value & $\begin{array}{l}m_{1 / 2} \\
2.500 \cdot 10^{2}\end{array}$ & Common gaugino mass (1) \\
\hline signmuValue & $\begin{array}{l}\operatorname{sgn}(\mu) \\
1.000\end{array}$ & Sign of bilinear $H_{1} H_{2}$ coupling (1) - (3) \\
\hline AoValue & $\begin{array}{l}A \\
-1.000 \cdot 10^{2}\end{array}$ & Unified trilinear coupling (1) \\
\hline lambdaValue & $\begin{array}{l}\Lambda \\
40 . \cdot 10^{3}\end{array}$ & Scale of soft SUSY breaking (2) \\
\hline mmessValue & $\begin{array}{l}M_{\text {mess }} \\
80 . \cdot 10^{3}\end{array}$ & Overall messenger scale (2) \\
\hline nmessValue & $\begin{array}{l}N_{5} \\
3 .\end{array}$ & Messenger index (2) \\
\hline m32Value & $\begin{array}{l}m_{3 / 2} \\
60 . \cdot 10^{3}\end{array}$ & Gravitino mass (3) \\
\hline MAValue & $\begin{array}{l}m_{A} \\
200\end{array}$ & $A^{0}$ Higgs boson mass (4) - (14) \\
\hline tanbetaValue & $\begin{array}{l}\tan \beta \\
10\end{array}$ & $v_{2} / v_{1}$ (1) - (15) \\
\hline muValue & $\begin{array}{l}\mu \\
1.5 \cdot 10^{3}\end{array}$ & Bilinear $\mathrm{H}_{1} \mathrm{H}_{2}$ coupling \\
\hline M1Value & $\begin{array}{l}M_{1} \\
300 .\end{array}$ & Gaugino soft breaking mass (16) - (17) \\
\hline M3Value & $\begin{array}{l}M_{3} \\
360 .\end{array}$ & Gaugino soft breaking mass 16 \\
\hline msleptonValue & $\begin{array}{l}m_{\tilde{l}} \\
400 .\end{array}$ & Common slepton breaking mass 17 \\
\hline xBFS2013Value & $\begin{array}{l}x \\
0\end{array}$ & Slope parameter $x$ \\
\hline yBFS2013Value & $\begin{array}{l}y \\
0 .\end{array}$ & Slope parameter $y$ \\
\hline muRenValue & $\begin{array}{l}\mu_{\text {ren }} \\
173.3\end{array}$ & Renormalization/output scale \\
\hline
\end{tabular}

The next set of options can be left at their default values, but can be changed if needed

- SQLVarCharLengthValue ->50

gives the maximum length of strings saved in the data base in the "VARCHAR" format.

- InputFilePathValue->

(Environment ["HOME"] <>"/.SLAM/tmp/LesHouches.in")

contains the name and location of temporary SLHA input files as a string.

- OutputFilePathValue->

(Environment ["HOME"] <> "/.SLAM/tmp/LesHouches . out")

contains the name and location of temporary SLHA output files as a string.

- The default values of the parameters are defined. All parameters and their default values are listed together with a brief description in Table 1. 
- After the default numerical values are set, one is able to configure the default input from SLAM for further processing. For example: LesHouchesInputRequest $=\{$ MASS" $->\{25->\{$ Mh0, Real $\}\}\}$;

will just return the lightest Higgs boson mass by default. A more general example for the list on the right side of the definition can be taken from Mbx. (m4).

- UseMinimalFlavourViolation=True;

Sets the used set of declarations done in the bottom of the configuration file to the minimal flavour violation one. If one works within minimal flavour violating models one should keep this setting. All the predefined scenarios work within this framework. If one works beyond minimal flavour violation, one may set the variable to False.

The following settings should only be changed by experienced users, who intend to extend SLAM.

- LesHouchesInputSQLFormats $=\{\ldots\}$

Contains declarations of input parameters, which are required by spectrum generators. These declarations are used to define the data table layout used in the SQL data base. Possible data types are String, Integer and Real.

A change of the declarations requires a reset of the used data base. SLAM requires a proper set of declarations in order to be able to use a particular data base table.

- LesHouchesOutputSQLFormats $=\{\ldots\}$

Contains declarations of output parameters, which are generated by spectrum generators. These declarations are used to define the data table layout used in the SQL data base. Possible data types are String, Integer and Real.

A change of the declarations requires a reset of the used data base. SLAM requires a proper set of declarations in order to be able to use a particular data base.

If one wants to load SLAM in a more convenient way with the command Needs ["SLAM' "]; the path to the folder where SLAM.m is located can be added to the list of folder names stored in the global \$Path variable. This can be done for example in the \%.Mathematica/Kernel/init.m file by the following command:

\$Path=Join [\$Path, $\{" /$ some/path/SLAM" $\}]$;

Concerning the installation and configuration of a SQL server, we recommend to follow the instructions given in the particular server manual. However, we want to add some comments about how to speed up the SQL server in the following.

The access to the data base can be greatly improved by adding a key or an index to the most important columns of the table. For example in the case of the $m_{h}^{\max }$ scenario the most obvious choice would be to at least add a key to the columns containing the input parameters $m_{A}$ and $\tan \beta$. For other scenarios a different choice might be more suitable. ${ }^{5}$ Keys can be defined directly after the initial declaration of the layout of the data base or added at any later time at almost no cost. To define a key using Mathematica considering for example the $m_{h}^{\max }$ scenario one can use the command

SQLExecute [\$LesHouchesSQLConnectionList [[1]], \#] \& / @

\{"alter table SPECTRATABLE add key (I_EXTPAR_26);" (*MA*),

"alter table SPECTRATABLE add key (I_MINPAR_3);" (*tanbeta*)\};

which requires an active data base connection stored in \$LesHouchesSQLConnectionList. An active data base connection can be obtained best by calling ObtainLesHouchesSpectrum [] with the option KeepSQLConnection set to True once, before executing the command above. Since the Mathematica function SQLExecute [] used in this example simply executes the given SQL command, one can easily read off the necessary SQL commands enclosed as strings. If one is interested in the inverse problem, i.e. looking up spectra which e.g. have a Higgs boson in a certain mass range, it is helpful to define a key for the respective column. For a definition of column names in the data base table see Section 5.3.

\section{Usage instructions}

In this section we try to give detailed instructions about how to use the SLAM package. Mainly this comes down to the knowledge how to use the function ObtainLesHouchesSpectrum. But also the built-in function ReadLesHouchesSpectrumFile might be of general interest because it can automatically import any SLHA file into Mathematica. The same holds for the function WriteLesHouchesFile, which automatically writes SLHA files according to the input provided in Mathematica.

\subsection{Using ObtainLesHouchesSpectrum}

The function ObtainLesHouchesSpectrum is completely controlled via options. One might even call the function without giving an explicit option:

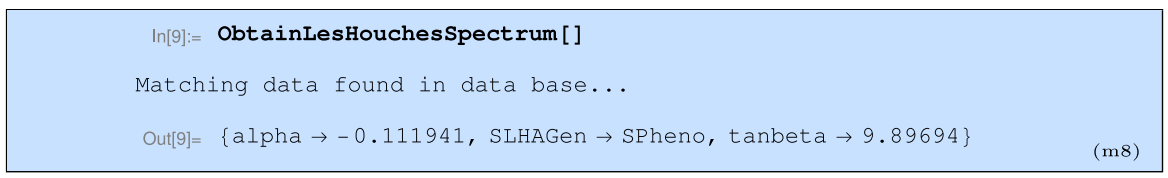

5 See Table 2 for examples of free scenario parameters. 
Table 2

List of all predefined models available in SLAM. Use the strings listed in the Option value column as option value for the Model in order to select the corresponding model. All free parameters of each model are shown in the last column.

\begin{tabular}{|c|c|c|c|}
\hline Model & Option value & ID/Ref. & Free parameters \\
\hline mSUGRA & "msugra" & (1) & $\begin{array}{l}\mathrm{m} 0, \mathrm{~m} 12, \mathrm{~A} 0 \\
\text { signmu, tanbeta }\end{array}$ \\
\hline mGMSB & "mgmsb" & (2) & $\begin{array}{l}\text { lambda, mmess, } \\
\text { tanbeta, signmu, nmess }\end{array}$ \\
\hline mAMSB & "mamsb" & (3) & m0, m32, tanbeta, signmu \\
\hline$m_{h}^{\max }$ & "mhmax" & (4) $[18]$ & MA, tanbeta \\
\hline no-mixing & "nomix" & (5) [18] & MA, tanbeta \\
\hline gluophobic & "gluophob" & (6) [18] & MA, tanbeta \\
\hline $\operatorname{small} \alpha_{\text {eff }}$ & "smallalpha" & (7) [18] & MA, tanbeta \\
\hline updated $m_{h}^{\max }$ & "mhmaxup" & (8) [21] & MA, tanbeta \\
\hline$m_{h}^{\bmod +}$ & "mhmod+" & (9) $[21]$ & MA, tanbeta \\
\hline$m_{h}^{\bmod -}$ & "mhmod-" & (1) $[21]$ & MA, tanbeta \\
\hline light stop & "lightstop" & (11) $[21]$ & MA, tanbeta \\
\hline light stau & "lightstau" & (12) $[21]$ & MA, tanbeta \\
\hline light stau $\left(\Delta_{\tau}\right)$ & "lightstaudeltatau" & (13) $[21]$ & MA, tanbeta \\
\hline$\tau$-phobic & "tauphobic" & (14) $[21]$ & MA, tanbeta \\
\hline low- $m_{h}$ & "lowmh" & (15) $[21]$ & $\mathrm{mu}$, tanbeta \\
\hline p19MSSM I & "p19MSSM1" & (16) [19] & M1, M3 \\
\hline p19MSSM II & "p19MSSM2" & (17) [19] & M1, mslepton \\
\hline 374345 & "374345" & (18) $[22]$ & \\
\hline 401479 & "401479" & (19) [22] & \\
\hline 1046838 & "1046838" & (2) [22] & \\
\hline 2342344 & "2342344" & (21) $[22]$ & xBFS2013, yBFS2013 \\
\hline 2387564 & "2387564" & (2) [22] & \\
\hline 2750334 & "2750334" & (23) [22] & \\
\hline
\end{tabular}

This calls ObtainLesHouchesSpectrum with all options set to their default values. A list of all options can be obtained using:

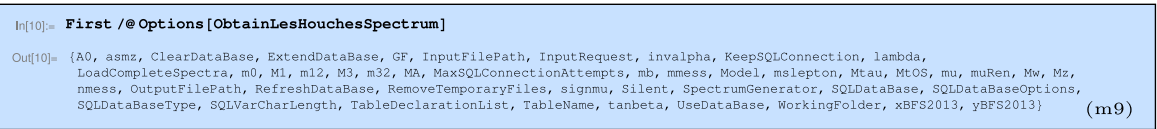

One can get further information about a single option by the question mark operation as follows:

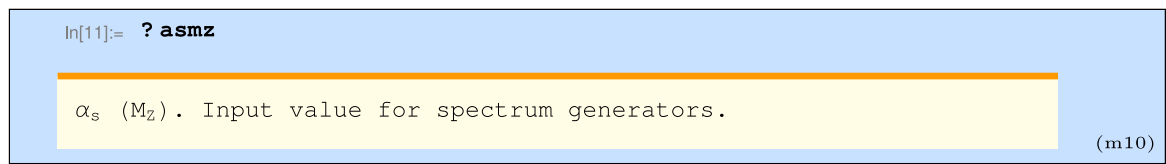

The default option value can be checked with:

$\ln [12]:=$ asmz /. Options [ObtainLesHouchesSpect rum]

Out[12] $=0.1184$

$(\mathrm{m} 11)$

and set via:

$\ln [13]:=$ SetOptions [ObtainLesHouchesSpectrum, asmz $\rightarrow 0.11835$ ];

In principle one can just go through all options using the given commands for each option in order to get to know all of them.

Since this may be tedious, we just pick out classes of options and discuss them step by step.

The first class of options is part of predefined scenarios. In detail they are just the free parameters left in the corresponding scenario and listed in Table 2. Removing these options from the list in Mbx. (m9) the number of unknown options reduces by 16.

The next class of options is built up by the SM parameters which need to be provided to the spectrum generators. They are listed in Table 3.

This further reduces the amount of unknown options by 9 .

The next class is built up by options used to steer the processing. A list of these options including their default values are given in Table 4. In the following we give more detailed information on each of these options:

- InputFilePath/OutputFilePath

The path including the name of SLHA input/output file as a string. 
Table 3

List of options used to specify SM parameters needed by the spectrum generators. All values are given in proper powers of $\mathrm{GeV}$.

\begin{tabular}{|c|c|c|}
\hline Variable & $\begin{array}{l}\text { Symbol } \\
\text { Default value }\end{array}$ & Description \\
\hline invalpha & $\begin{array}{l}1 / \alpha^{\overline{M S}}\left(m_{Z}\right) \\
1.279340000 \cdot 10^{2}\end{array}$ & Inverse fine structure constant \\
\hline GF & $\begin{array}{l}G_{F} \\
1.16637 \cdot 10^{-5}\end{array}$ & Fermi-constant \\
\hline asmz & $\begin{array}{l}\alpha_{s}^{(5), \overline{M S}}\left(m_{Z}\right) \\
0.1184\end{array}$ & QCD fine structure constant \\
\hline Mw & $\begin{array}{l}m_{W} \\
80.399\end{array}$ & $W$ boson mass \\
\hline $\mathrm{Mz}$ & $\begin{array}{l}m_{Z} \\
91.200\end{array}$ & $Z$ boson mass \\
\hline $\mathrm{mb}$ & $\begin{array}{l}m_{b}^{\overline{M S}}\left(m_{b}\right) \\
4.25\end{array}$ & $b$ quark mass \\
\hline MtOS & $\begin{array}{l}M_{t} \\
173.3\end{array}$ & $t$ quark pole mass \\
\hline Mtau & $\begin{array}{l}m_{\tau} \\
1.777\end{array}$ & $\tau$ lepton pole mass \\
\hline muRen & $\begin{array}{l}\mu_{\text {ren }} \\
173.3\end{array}$ & Renormalization/output scale \\
\hline
\end{tabular}

Table 4

List of options used to control the core operation of SLAM.

\begin{tabular}{ll}
\hline Option & Default value \\
\hline InputFilePath & see InputFilePathValue \\
InputRequest & see Mbx. (m4) \\
Model & "msugra" \\
OutputFilePath & see OutputFilePathValue \\
RemoveTemporaryFiles & True \\
Silent & False \\
SpectrumGenerator & "spheno" \\
WorkingFolder & Automatic $\rightarrow$ folder of InputFilePath \\
\hline
\end{tabular}

- InputRequest

Defines the data, which should be returned:

$-\mathrm{All}$

returns everything that is available in an internal SLHA format, built up from lists and replacement rules. For more details about the used format see Section 5.2 .

In fact the output still depends on the source of the data:

If SLAM got the data from a SLHA-file written by a generator, the entry values are still kept as strings, even though they are integers or real numbers, because SLAM does not know, which format transformation should be applied.

If SLAM loaded the data from the data base, all entry values will have their proper format.

- AllFormated

Returns only blocks of entry values, which were declared in SLAM. config.m. Moreover SLAM tries to convert all data to the proper format contained in the declaration.

- Direct INPUT

Can be used to handle direct input, which selects certain data to be returned as a list of replacement rules, where the symbol of the left hand side of each rule can be freely chosen. Examples for such selections can be found in Section 2.1 in Mbx. (m3). The default value for this option is printed in Mbx. ( $\mathrm{m} 4)$, where only entry values (EV) are selected. It is also possible to select block comments $(\mathrm{BC})$, entry value comments (EC) and block information (BI) as follows:

$$
\begin{aligned}
& \text { \{"MASS" } \rightarrow \text { BBC } \rightarrow \text { \{MASSComment, String\}, } \\
& \{25, E C\} \rightarrow\{\text { MhOComment, String }\} \text {, } \\
& \text { "MSOFT" }>\text { \{BI } \quad->\text { muRen, Real }\}\} \text {; }
\end{aligned}
$$

Note that if data is requested, that was not declared in the data base table, SLAM will print a warning and abort the process, if the data base is in use. If this is not the case, the data will be returned as \$MissingData in case the data is missing.

- WorkingFolder

determines the place where temporary files for and from spectrum generators are created. If one keeps the default option value, given by Automatic, SLAM will extract the directory from the InputFilePath option and use it as a working folder. 
Table 5

List of options used to control the data base operations of SLAM.

\begin{tabular}{ll}
\hline Option & Default value \\
\hline ClearDataBase & False \\
ExtendDataBase & True \\
KeepSQLConnection & True \\
LoadCompleteSpectra & False \\
MaxSQLConnectionAttempts & 10 \\
RefreshDataBase & False \\
SQLDataBase & see SQLDataBaseValue \\
SQLDataBaseOptions & see SQLDataBaseOptionsValue \\
SQLDataBaseType & see SQLDataBaseTypeValue \\
SQLVarCharLength & see SQLVarCharLengthValue \\
TableDeclarationList & see Section 5.3 \\
TableName & "SPECTRATABLE" \\
UseDataBase & True \\
\hline
\end{tabular}

- Model

selects either between different predefined scenarios indicated by a special string or allows direct input, specifying the scenario via an internal SLHA format.

- "..."

For predefined scenarios all possible choices of allowed strings can be found in Table 2.

- Direct INPUT

A minimal example for the direct, or user-defined model input, is given in Section 2.4. For a better understanding of the used format the user may be referred to Section 5.2. The format enables the user to add block comments or block information, or even entry comments which will be written to the SLHA input file and saved in the data base. However, it is very important that all values should be given in their proper format. That means, if one wants to provide an integer it needs to have the Head Integer. If one wants to provide a floating point number, the Head of the number should return Real. Even if the number happens to be an integer one should append a dot to make its Head a Real. This is because SLAM has to convert the given input to proper FORTRAN readable strings. In case one already has a proper SLHA input file, it is possible to automatically generate the direct input using the ReadLesHouchesSpectrumFile function (see Section 4.4 for more details), in order to avoid time-consuming retyping.

- RemoveTemporaryFiles

can be True or False. When set to True SLAM deletes all temporary generated files automatically after reading them. When set to False they are kept.

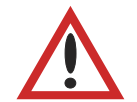

When set to False one can easily get wrong results because SLAM may just load an old file in the case where no new file was generated by a spectrum generator. Thus this setting should only be used when one wants to have a look into the files.

- Silent

- "True"

Will stop SLAM to print processing messages.

- "False"

Processing messages get printed.

- SpectrumGenerator

Specifies the spectrum generator that should be used. Currently the following generators can be used, when installed and configured correctly:

- "spheno",

- "softsusy",

- "suseflav",

- "suspect".

With the given options one can fully control the call of spectra from generators. However, the options steering the communication with a data base are still missing. They constitute the last class of options which will be introduced now. In Table 5 all options can be found in alphabetic order, including their default values and are discussed in this order:

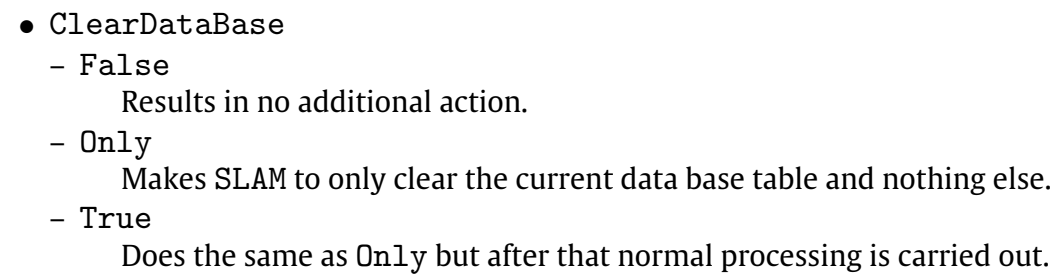

Clearing a data base table leads to a complete loss of all stored data. Use this option carefully! 
- ExtendDataBase

- False

- True

New spectra are not saved in the data base.

New spectra are saved in the data base.

- KeepSQLConnection

- False

- True

Makes SLAM disconnect from the SQL server after every transaction.

Makes SLAM to hold the connection to the SQL server throughout a Mathematica session.

This setting may be useful when one has more than one Mathematica kernel running SLAM at the same time (parallel) but using only one SQL server. Depending on the SQL server settings, only a maximum amount of clients may be allowed to connect to the server. That means holding the connection without need may potentially block another client from getting its data. However, establishing and closing a connection after every interaction may increase the network traffic to an unacceptable amount.

- LoadCompleteSpectra

- True

Makes SLAM to load all data values contained in one spectrum and stored inside the data base table, although the user might only have requested some of the data values. However, only the requested data is returned after a filtering.

- False

Makes SLAM to load only the requested data values from the data base. This requires some internal transformation of the InputRequest data to a SQL readable form, which might slow down the process for large requested sets of data. However, with this setting the network traffic is reduced in any case and for a small number of requested data values this option leads to much faster loading times.

One should keep the default value (False) in case of a small number of data values in a request. For requests close to a full data set the option True might speed up the calculation because there is no processing time going into the conversion from InputRequest data to a SQL readable form.

- MaxSQLConnectionAttempts

Can be any positive integer and sets the total amount of connection attempts to an SQL server, until SLAM stops the operation throwing an error message.

- RefreshDataBase

- False

- True

Makes SLAM to load any matching spectra from the data base.

Makes SLAM to overwrite (refresh) matching spectra in the data base with the data of a newly generated spectrum.

- SQLDataBase

Contains the address of the used data base as a string. In case the Mathematica internal data base is used, this string is given by the system path where Mathematica will write its data base files to. See SQLDataBaseValue for examples.

- SQLDataBaseOptions

Contains a list of options which specify mainly the user account on the used data base. See SQLDataBaseOptionsValue for examples.

- SQLDataBaseType

Contains a string defining the kind of data base in use. See SQLDataBaseTypeValue for examples.

- SQLVarCharLength

Gives the maximum number of characters contained in a string saved to the data base as VARCHAR. This option is only in use when a new data base is being created.

- TableDeclarationList

Gives a list of declarations for the data base. The default value is generated automatically from the settings given in the file SLAM. config.m when the SLAM package gets loaded. One should not use any option other than default, except for testing reasons. See Section 5.3 for more details.

- TableName

Holds a string which gives the name of the table inside the data base where all the spectra data are stored. Note that one might use different table names for different scenarios for convenience, especially for later manual analysis which may be independent of SLAM. However, SLAM can distinguish different scenarios on its own, so saving spectra obtained within different scenarios does not lead to wrong results.

- UseDataBase

- False

Forbids SLAM to use the data base. Thus any spectrum request has to be served by a spectrum generator call. - True

Allows SLAM to use the data base. This means any matching spectrum found in the data base will be used, instead of calling a spectrum generator.

We have now described all relevant options, we need to state some important usage restriction:

Although the SLHA allows for the printout of scale dependent parameters at different scales $Q$ in a single SLHA file, SLAM is not able to treat multiple appearances of a block at different scales. That means one should use the spectrum generators in user-defined scenarios in such a way, that each block is appearing only once per SLHA file.

If one needs parameters at different scales, the spectra have to be called separately for each scale.

Since we have now all the basics at hand, we can show some more involved applications of our package. 


\subsection{Advanced example}

A nice example for the comfortable usage is the calculation of the light Higgs boson mass in a scenario depending on two parameters, like the $m_{h}^{\max }$ scenario. How easy it is to get the relevant data shows the following code:

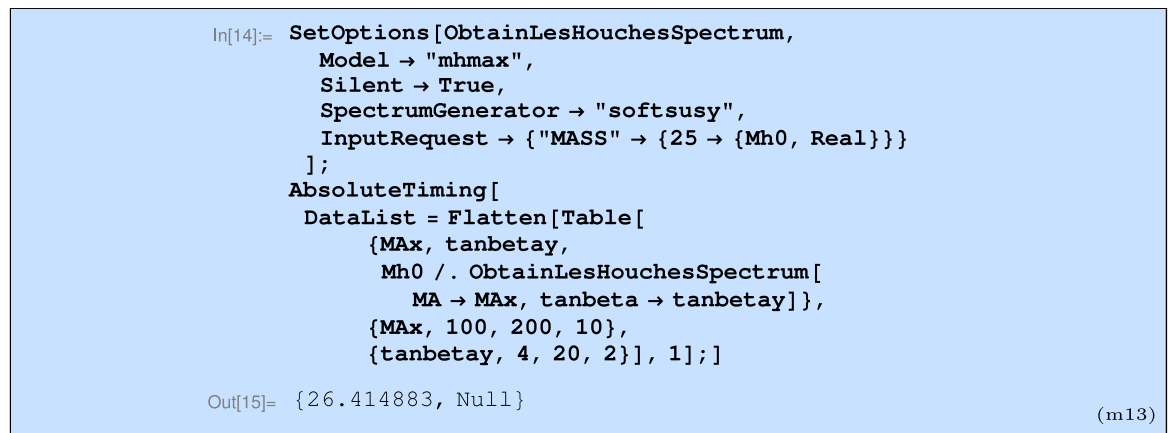

With the first command the default options of ObtainLesHouchesSpectrum get changed. We select the $m_{h}^{\text {max }}$-scenario, make the calculation silent, which means printouts are disabled, chose SOFTSUSY as the spectrum generator and request only the light Higgs boson mass to be in the output. Changing the default values has the advantage, that we do not need to specify the options in the call of ObtainLesHouchesSpectrum itself, which keeps the next command clear and short.

In the second command, we generate the Higgs mass data in dependence of the two free parameters $M_{A}$ and tan $\beta$. This is done with the help of a simple Table function, which automatically creates all possible combinations of $M_{A}$ and $\tan \beta$ values in the stated limits. Note that for each combination ObtainLesHouchesSpectrum is called with the current value of the two parameters. Since this function returns just a replacement rule for Mh0, one has to use the called function like a replacement rule acting on the symbol Mh0. The next to outer-most Flatten function reduces the structure to a list of lists, where each of the lists contains three elements: the x-value $M_{A}$, the $\mathrm{y}$-value $\tan \beta$ and the $\mathrm{z}$-value $M_{h^{0}}$. The outer-most AbsoluteTiming function just returns the absolute calculation time in seconds of the command in its argument, which was about $26 \mathrm{~s}$, using the Mathematica built-in data base. This runtime was consumed to run the spectrum generator and fill the data base with data from 99 spectra.

It is straightforward to display the calculated data, e.g. the following code does this job in a minimal way:

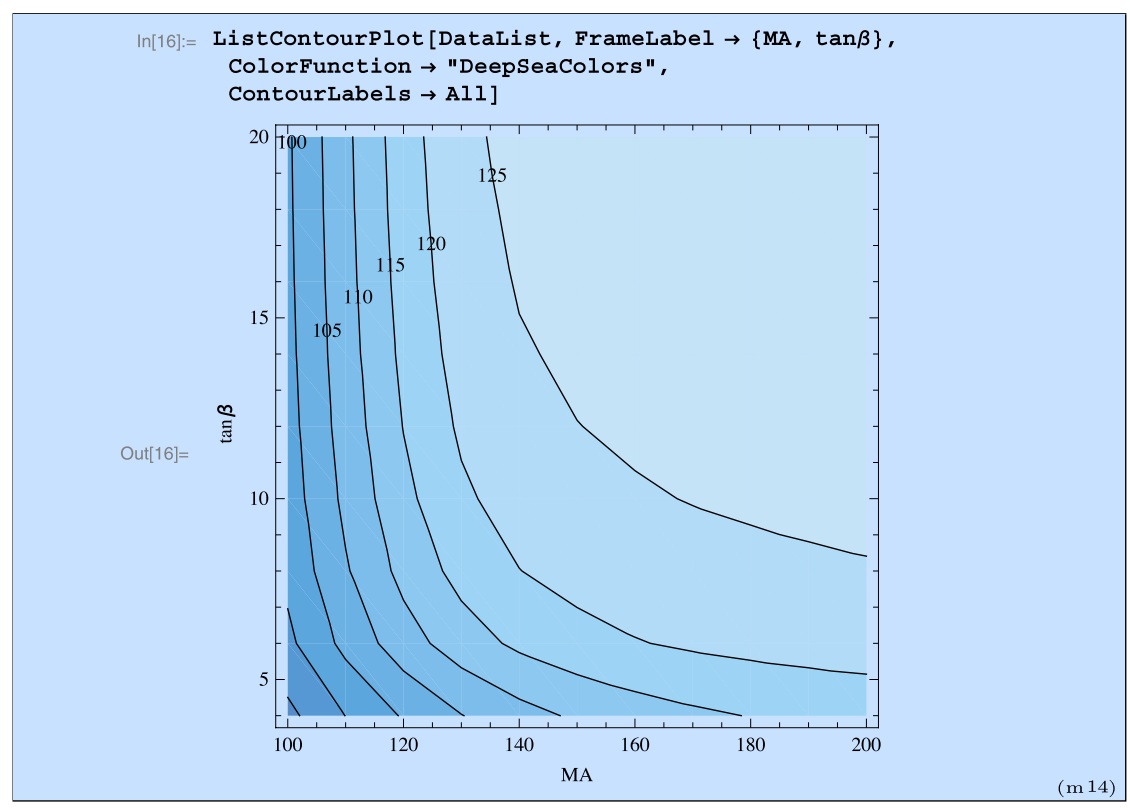

The plot shows the Higgs mass in GeV in dependence of $M_{A}$ and $\tan \beta$.

In this example we started with an empty data base and the spectrum generator had to run for every combination of input values. We can rerun the command with the already populated data base

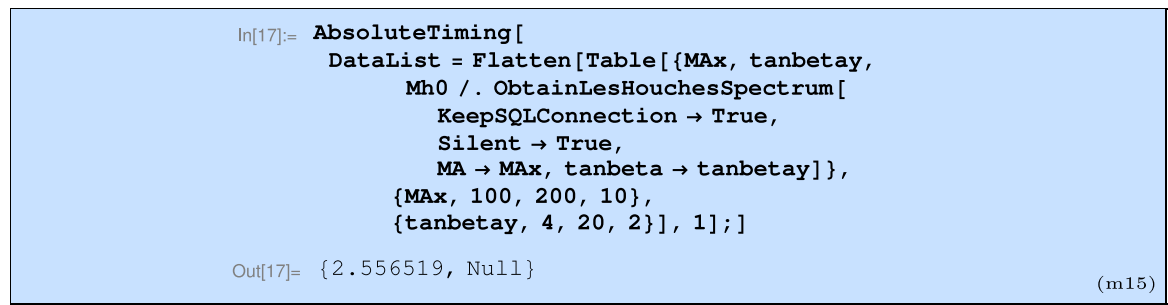

and find that the processing time now is about ten times smaller. 
Table 6

List of all options of the function ReadLesHouchesSpectrumFile. ${ }^{\dagger}$ defined in SLAM. config.m.

\begin{tabular}{ll}
\hline Option & Default value \\
\hline AdoptEntryFormats & True \\
DataStructure & LesHouchesOutputSQLFormats ${ }^{\dagger}$ \\
Input & File \\
InputRequest & LesHouchesInputRequest ${ }^{\dagger}$ \\
RemoveTemporaryFiles & True \\
OutputFilePath & OutputFilePathValue ${ }^{\dagger}$ \\
\hline
\end{tabular}

\subsection{Using WriteLesHouchesFile}

The function WriteLesHouchesFile is normally called by ObtainLesHouchesSpectrum, but because it might be useful to be able to write SLHA files directly from Mathematica and independent of the spectrum generator usage, this function can be called independently. The function has only three options, which shall be discussed now:

- InputFilePath

Holds a string which contains the path and the name of the file.

- InputList

Contains the actual data that gets written to the file. An example for the formatting of the data can be displayed with:

InputList/.Options [WriteLesHouchesFile]

Further information about the format can be found in Section 5.2.

- AppendToFile

- False

WriteLesHouchesFile clears already existing content in the target file before writing output to it.

- True

WriteLesHouchesFile appends its output after already existing content in the target file.

With the given function one can write arbitrary data to a SLHA file, as the following example shows:

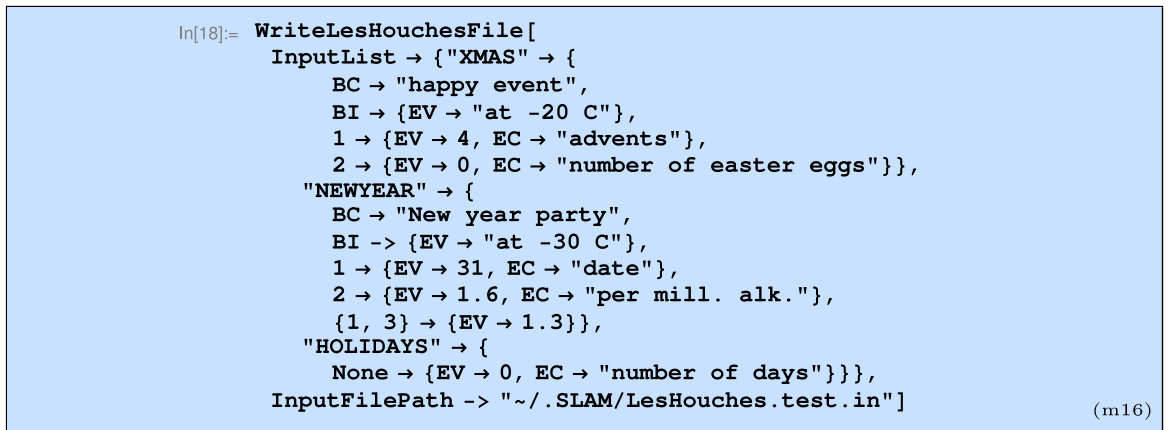

This example leads to the output file:

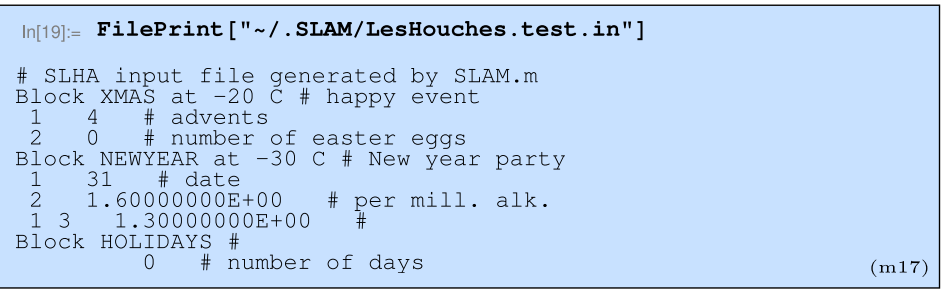

This shows that the application of WriteLesHouchesFile is not restricted to the spectrum generator interface. Note that block information (BI), block comments (BC) and entry value comments (EC) are optional in the InputList. Note further that WriteLesHouchesFile automatically detects the format of the given entry value (EV). All other values and comments have to be strings.

\subsection{Using ReadLesHouchesSpectrumFile}

The function ReadLesHouchesSpectrumFile, like its name suggests, reads in SLHA files. It has several options which are listed in alphabetic order including their default values in Table 6. The options and their descriptions are:

\footnotetext{
- AdoptEntryFormats
}

- False

Will keep the string format of all entry values read in. 
- True

Will convert the entry format according to either InputRequest or DataStructure, depending on the value of InputRequest.

- DataStructure

Gives the default declaration of all entry values. Its default value is given by LesHouchesOutputSQLFormats in the SLAM. config.m file. Note, that this can be changed to what is required for the content of the SLHA file getting loaded.

- Input

- File

Tells ReadLesHouchesSpectrumFile to read in the file according to the path and the file name provided in OutputFilePath.

- Direct string input

Will be read like stemming from a file.

- InputRequest

- All

returns everything without touching the data format, that means everything stays a string.

- AllFormated

Returns only blocks of entry values which are declared in the DataStructure option. Further, all values are converted to their declaration formats, if AdoptEntryFormats is set to True. Use this option value in order to generate direct input useable as Model option value of ObtainLesHouchesSpectrum.

- Direct Input

Only the selected input values will be returned in a replacement rule, working like for ObtainLesHouchesSpectrum.

- RemoveTemporaryFiles

- True

Tells ReadLesHouchesSpectrumFile to delete the file after reading it.

- False

Will keep the file unchanged after reading it.

- OutputFilePath

Provides a string holding the path and the name of the file that should be read.

Because we already have written out an example SLHA file in the previous section, we can give an usage example of the function ReadLesHouchesSpectrumFile by just loading in, what we have written out:

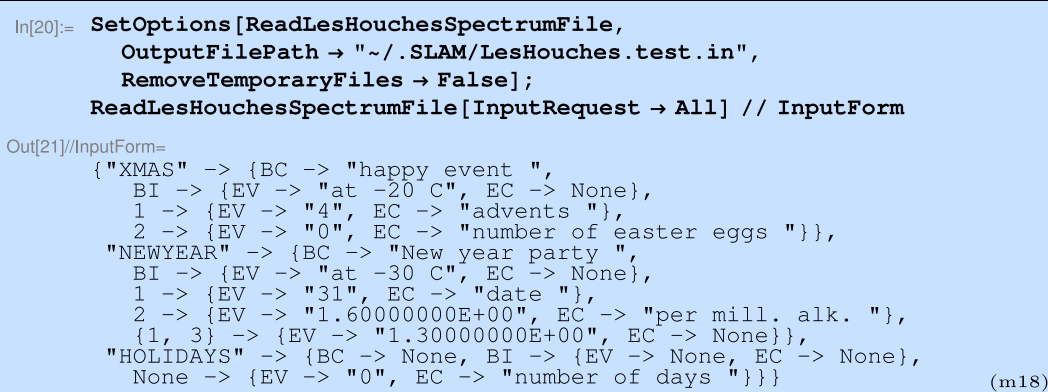

In the code above we set the default value of RemoveTemporaryFiles to False to be sure that we can load in the input file again. Further we specify the file which should be loaded. After that we call the ReadLesHouchesSpectrumFile function, requesting everything that can be found in the SLHA file by setting the option InputRequest - $>$ All. We show the output in the InputForm to be able to distinguish strings from regular symbol names. The output shows everything that is contained in the file, but the entry values (EV) are kept as strings.

In order to adopt their original format, we can load the file with the option InputRequest - >AllFormated. Because the default option of DataStructure, which is given by LesHouchesOutputSQLFormats, does not contain any suitable declaration information for our little toy example, we have to provide the proper declaration through the DataStructure option on our own. This is done in the following:

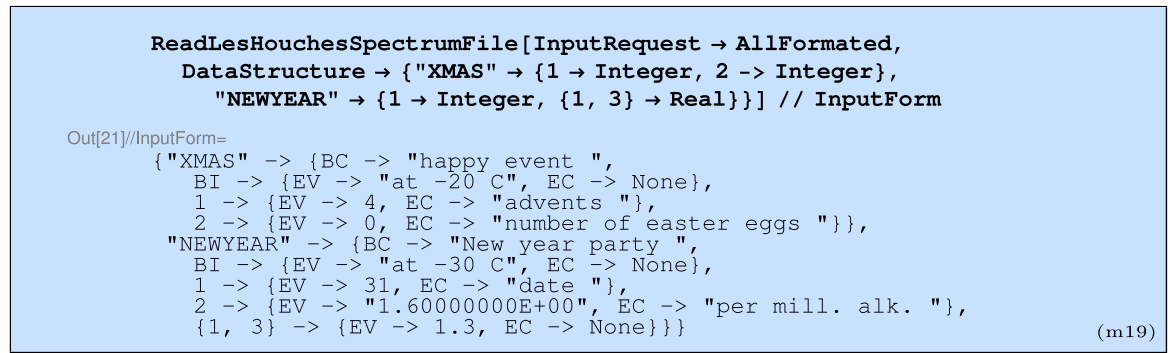

Compared to the output of Mbx. (m18), we see that all entry values which have been declared got converted from the string to the declared format. Since we did not declare anything for the block "HOLIDAYS", this block is completely missing. 
In the last example we directly request just the number of advents in block "XMAS":

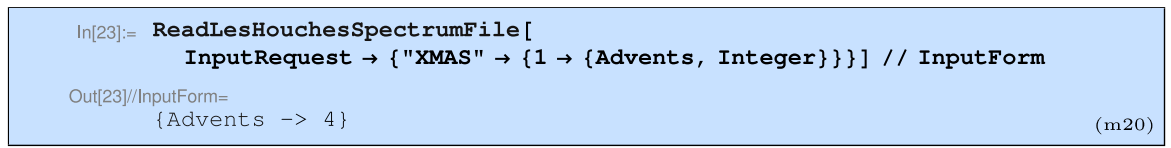

This results in a list containing a replacement rule, where our choice of symbol for the number of advents has been taken over and the number was properly converted to an integer.

\section{Package internals}

In this section we provide information, which enables the user to extend or adjust SLAM to very special needs. Before going into details, some words about the general layout of the package and the spirit in which it has been programmed are in order.

The package is purely written in Mathematica. Only very little bash code is used to manage the spectrum generators and the SLHA files. Throughout the package the code was written in a modular way, heavily relying on the built-in options system of Mathematica. A comprehensive introduction to the options interface can be found in the Mathemati ca help centre. It is easy to write argument insensitive code for the call of a sub function inside a normal function, if one uses the options interface. One does not need to worry about which argument has to be put at which position and it is no problem to add further parameters later, without changing the code for the function call itself. With this feature one can conveniently write modular code, grouping different tasks into different, topic oriented functions. This helps to keep the code local and modular, which is very convenient while debugging. If something is not working properly, one can just check the data exchanged between modules. Finding the place where wrong data appears leads directly to the faulty module. Moreover, since every module serves a very special purpose only, it is simpler to ensure that each module fulfils its task properly than just writing a single code which has to achieve many goals properly at the same time. Thus following the old maxim "divide et impera", 6 one can write complicated code achieving multiple goals properly by just writing small modules doing their job properly and finally connecting all of them.

In the following subsection we are going to give a "map" of all relevant main modules which work inside the ObtainLesHouchesSpectrum function. We will walk on this map through the steps which are performed automatically by Mathematica in order to carry out commands given by the user. During this subsection the reader should get a basic orientation, needed for any modification of the code.

The second subsection clarifies the Mathematica internal representation of the SLHA used in this package.

In the third subsection we explain the used layout of the data base table in more detail.

The fourth subsection should enable the reader to implement new predefined scenarios in the existing code of SLAM.

\subsection{Internal structure of ObtainL esHouchesSpectrum}

In Fig. 1 a simplified "blueprint" of the function ObtainLesHouchesSpectrum is shown. The function connects a spectrum generator on the left and a SQL data base on the right with the output of the function at the bottom right, in dependence of input provided by the user, shown at the top left. Inside the function ObtainLesHouchesSpectrum, which is indicated by the light grey background, sub functions are displayed in white rectangular boxes. They are connected by red lines indicating the order of sub-function calls. The yellow diamonds represent branchings of function calls in dependence of certain conditions. The rounded boxes stand for data prepared in a certain format indicated by the colour of the background. In fact all names inside are actual function and data names used in the Mathematica code. Black arrows show the flow of data.

In the following the flowchart is described step by step. The starting point of this description is the place where the user input enters ObtainLesHouchesSpectrum:

1. In dependence of the option ClearDataBase the SQL data base table will be removed by the sub function DropSQLLesHouchesTable when True or Only.

2. In case of Only the evaluation ends after that. $\otimes$

3. When True or False was selected it generates the data GeneratorInputSQLDefinitions with either the

ToSQLLesHouchesData or the GenerateInputSQLChecklist function. The first one is used in case a direct input model is used, the second function will do the job when a predefined model is considered.

4. When the option UseDataBase is True, the next step is to check if there is already any data consistent with the defined Generator InputSQLDefinitions in the data base. This is done by calling the sub function SelectSQLLesHouchesTable.

(a) If there is any spectrum ready, it is stored in SQLDataBaseOutput which gets filtered by the sub function FilterLesHouchesBlockToInputRequest in order to provide the final output and end the evaluation. $\otimes$

(b) In case no proper data base table could be found it is created by the sub function CreateSQLLesHouchesDataBase. Further steps coincide with the case below.

(c) If no match was found, the spectrum needs to be obtained from the spectrum generator.

5. In order to obtain a spectrum from the generator, the proper SLHA input file has to be created. This is either done with the sub function WriteLesHouchesFile, when a direct input model is considered, or with the sub function WriteSpectrumGeneratorInput, when the predefined model is in use.

6. Then the spectrum generator is run by the sub function RunSpectrumGenerator.

6 lat. for "divide and rule" 


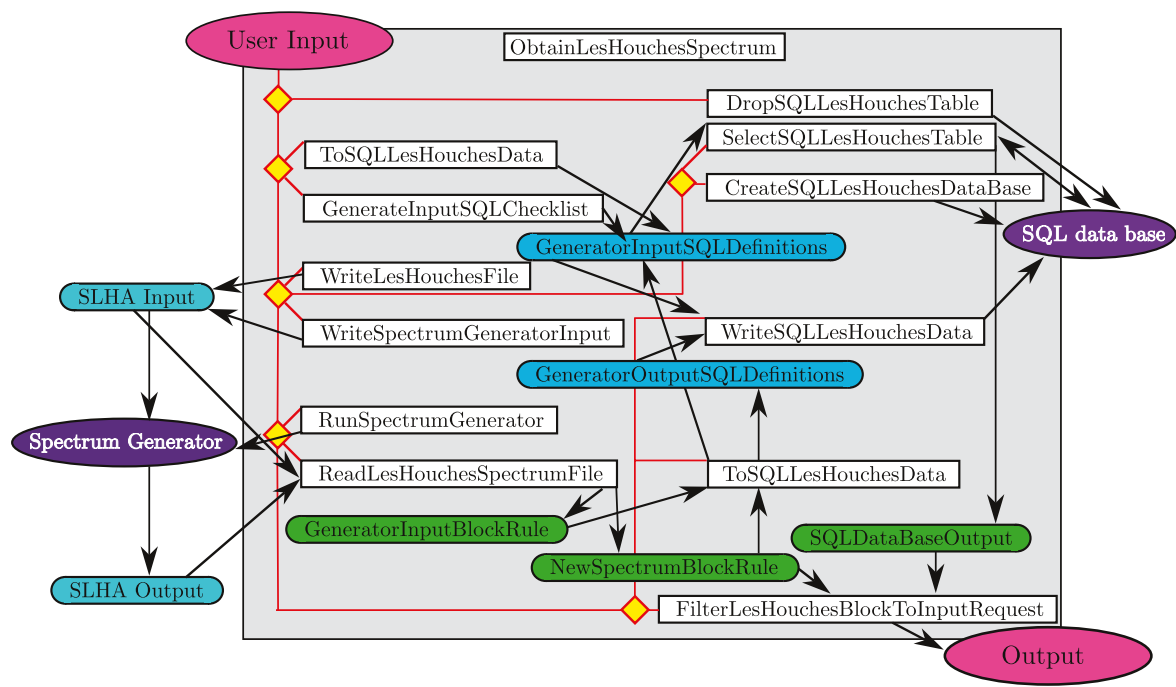

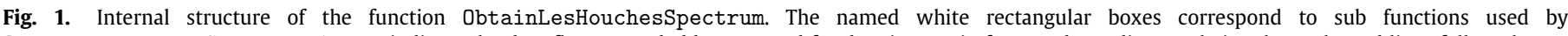

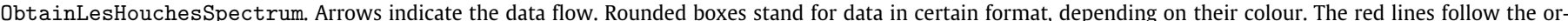

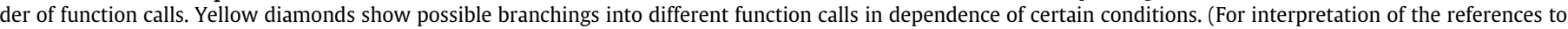
colour in this figure legend, the reader is referred to the web version of this article.)

7. If there was no problem in the previous step, the sub function ReadLesHouchesSpectrumFile is used to read in the SLHA output file and store the data in the expression NewSpectrumBlockRule.

8. In case the options ExtendDataBase and UseDataBase were set to True:

8.1 ReadLesHouchesSpectrumFile is used to read in the SLHA input file and store the data in the expressions GeneratorInputBlockRule.

8.2 ToSQLLesHouchesData transforms the data in GeneratorInputBlockRule and NewSpectrumBlockRule to the data base writable expressions GeneratorInputSQLDef initions and GeneratorOutputSQLDefinitions.

8.3 Then both sets of data are saved together in the SQL data base through the sub function WriteSQLLesHouchesData.

9. The final step is done by the sub function FilterLesHouchesBlockToInputRequest filtering the requested output from the data of NewSpectrumBlockRule in order to return it as output. $\otimes$

We did not discuss any option relevant for the sub functions. For example, the SpectrumGenerator option, telling ObtainLesHouchesSpectrum which spectrum generator should be used, does not appear in the code of the function at all. This is because ObtainLesHouchesSpectrum just trades these options to its sub function via a generic interface. This keeps the structure of the code very simple and generic.

\subsection{Internal representation for the SLHA}

In the following subsection we explain the Mathematica internal format of SLHA data, which is in use for the expressions GeneratorInputBlockRule, NewSpectrumBlockRule and SQLDataBaseOutput (see Fig. 1). The easiest way of explaining a certain format is to give just a data example. A SLHA output like:

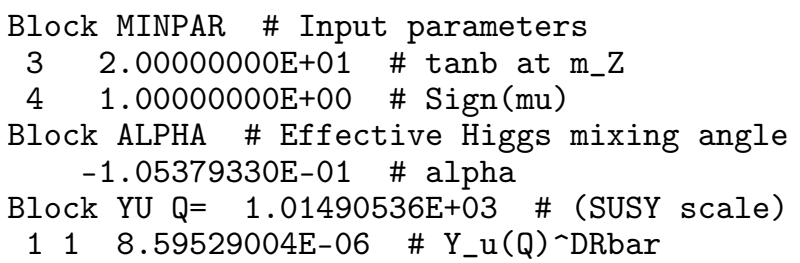

looks like the following in the Mathematica internal notation:

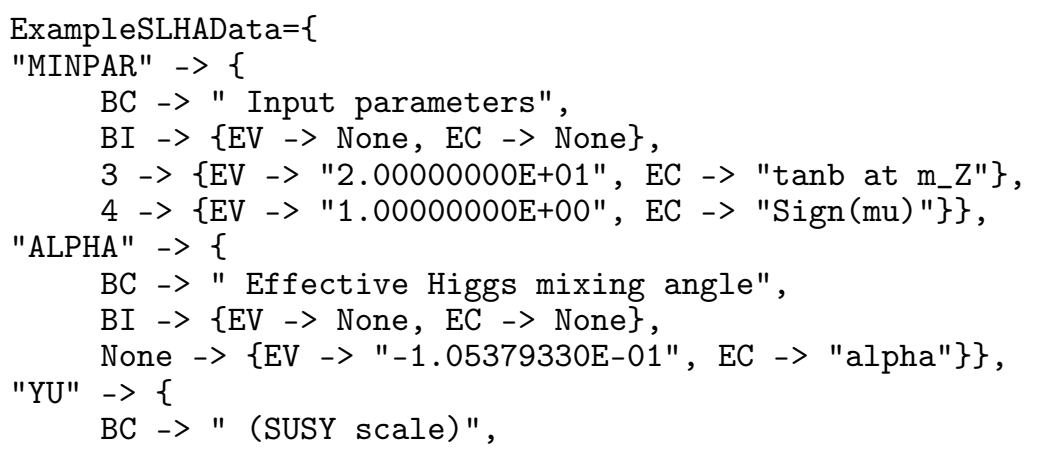




$$
\begin{aligned}
& \mathrm{BI} \rightarrow\{\mathrm{EV} \rightarrow \mathrm{Q}=1.01490536 \mathrm{E}+03 " \text {, EC } \rightarrow \text { None }\} \text {, } \\
& \{1,1\} \rightarrow\{\mathrm{EV} \rightarrow>\text { "8.59529004E-06", EC } \rightarrow>\text { "Y_u(Q)^DRbar" }\}\}\} \text {; }
\end{aligned}
$$

As one can see, the SLHA data is ordered with respect to blocks, where each block is just a replacement rule giving the block name in capital letters as a string on the left hand side of the rule and the content of the block in a list on the right hand side.

Each of those lists contains a replacement rule for the block comment (BC). Further it contains a replacement rule for the block information (BI), which has a list of two replacements on its right hand side. The latter contains a replacement for its entry value (EV) and entry comment (EC), where in case of the block information the last one is always None.

In case the entry value is given by a matrix element depending on two entry values, instead of a single integer as the entry key, a list of two integers is used, as can be seen in the last line.

In case where there is no entry key for a value, like for the mixing angle $\alpha$, the variable None is used as the entry key.

Note that in the example above all entry values are kept as strings. This only happens when SLHA files are loaded from a generator using the option InputRequest $->\mathrm{All}$.

In case of InputRequest - $>$ AllFormated SLAM tries to return converted entry values (e.g. real numbers) using the declarations made in the file SLAM. config.m.

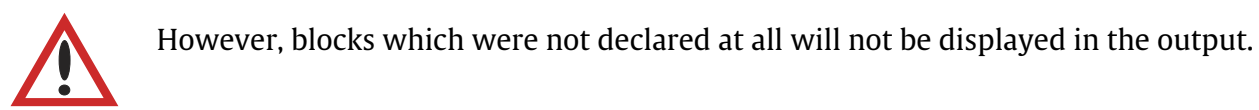

Entry values are returned in their declared format when loading spectra from the data base.

\subsection{Data Base Table Layout}

Because one can use the automatically created and built-up data base independently from SLAM, the layout of the table should be clarified. The default option value for TableName leads to the creation of a table with name "SPECTRATABLE".

Any table is declared and initialized with the information provided in the variables LesHouchesInputSQLFormats and LesHouchesOutputSQLFormats defined in the file SLAM.config.m. These variables are read in and the contained information is processed once, when the SLAM Package gets loaded, to give the full declaration list of the table. The full list of declarations can be printed using the command:

(TableDeclarationList /. Options [ObtainLesHouchesSpectrum]) // TableForm

We do not give the full default output here because it is quite lengthy, but pick only a selection for demonstration:

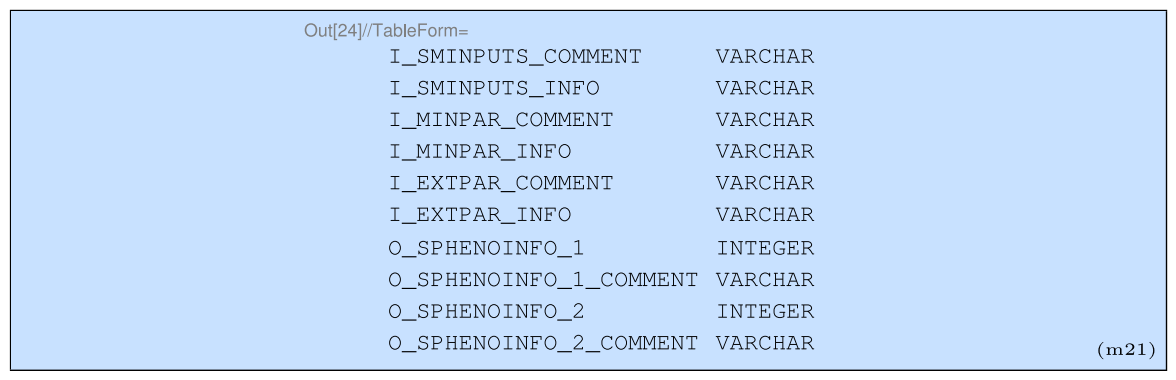

Note that all terms appearing in this output are in fact strings, because TableForm does not display the " character.

The output shows pairs of strings where the first string gives the name of the corresponding column and encodes mainly the place where the data is located in the SLHA file. The second string defines the type of data which will be stored in the corresponding column. So far there are only three different data types in use: "INTEGER", "DOUBLE" and "VARCHAR".

In the selection shown in Mbx. (m21) one can distinguish two different kinds of column names:

- Column names starting with a "I" reserve space for data stemming from SLHA input files.

- Column names starting with a "O" reserve space for data generated by the spectrum generators, so stemming from the SLHA output files.

Further the column names ending with COMMENT lead to columns, which do only save comments in the "VARCHAR" format. This is because one can optionally place comments behind the hash symbol (\#) in a SLHA file in each line.

After the initial "I" or "O" comes a block name in capital letters separated by an underscore.

- For columns which do not save a string one of the following holds then:

(a) At least one further underscore separated integer value is required. This value corresponds to the key value defined in the SLHA. See lines seven and nine in the displayed output above for example.

(b) If the value represents a matrix element there may be two underscore separated integers. Those integers correspond to two valued key entries defined in the SLHA.

(c) If the corresponding SLHA quantity has no key value, that means it comes alone in its own data block, like it is the case for the mixing angle $\alpha$, the string NONE has to follow the block name after one underscore.

- Every data value entry can have its own comment. The name of the comment column is obtained by simply appending the string _COMMENT to the name of the corresponding data value column. See lines ten and eight in the output above for example.

- Every block can have its own comment which is saved in the column name given by the block location appending the string _COMMENT. Lines one, three and five of the displayed output above give examples for this case. 
- Every block can have an additional information entry which is mainly used to hold the scale information. For example a block definition in a SLHA file might look like the following:

BLOCK GAUGE Q= 1.01490536E+03 \# (SUSY scale)

Everything after the space behind GAUGE and before the hash symbol (\#) gets stored in the block information (BI). The information is automatically saved in the "VARCHAR" format, as can be seen e.g. from lines two, four and six in the printout above where the name of the column is ending with the string _INFO. Note that requesting this data in the output as "Real" will force SLAM to return just the real number without the $Q=$.

With the given information it is easy to search directly in the data base for certain parameter limits. An example is to search for Higgs boson masses between $123 \mathrm{GeV}$ and $129 \mathrm{GeV}$ :

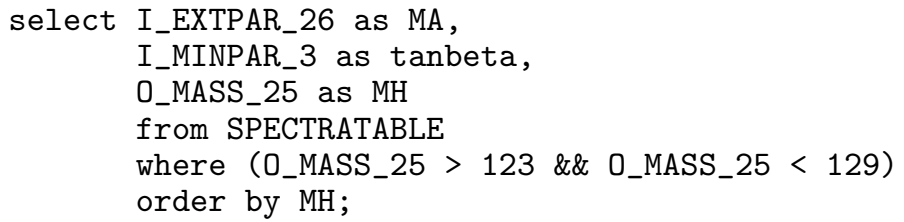

\subsection{Creating additional predefined scenarios}

Adding a new predefined scenario requires 6 steps but may help to speed up the generation of spectra, because less calculation steps have to be done for predefined scenarios in Mathematica.

1. If the new predefined scenario requires new option values because it depends on parameters which have not been used yet, one should add a new usage instruction before the Private section in the file SLAM . m for every symbol which will become an option. For example, the usage instruction for the nmess parameter used in the mGMSB-scenario looks like:

nmess: : usage="Number of minimal copies of the messenger sector in the gauge mediated symmetry breaking model.";

This enables the user to get information for that option via a question mark operation like: ?nmess.

2. Add the option name and description of the new scenario to the usage instruction of the Model variable:

Model : : usage=" ..."

For example the additional line should look like:

$\backslash$ "mgmsb\" (gauge mediated symmetry breaking).

3. Add the definitions for the default option value symbols related to the new option value in the SLAM. conf ig.m file. The names of the default option value symbols are just obtained from the option name itself by appending Value. See for example the nmessValue in Table 1. That way, the file SLAM.m will stay clean of any default numerical value definition and any user has a nice collection of the default numerical values in the config file.

4. Add the new options to the options of WriteSpectrumGeneratorInput. For our nmess example the corresponding line, which would have to be added, looks like:

nmess -> Global 'nmessValue,

5. The most difficult part is to add the new scenario consistently to the subfunction WriteSpectrumGeneratorInput. There are multiple Switch instructions where the new code for the new scenario has to be added. This can be done by just following the given examples in the code itself. Once having finished the modification of this function, one should check, after reloading the package, that the string written to the input SLHA file is correct when calling the new scenario. An easy way to do that is to call the modified function directly via:

SLAM'Private'WriteSpectrumGeneratorInput [Model -> "mgmsb", InputFilePath $\rightarrow$ String]

where one of course has to replace "mgmsb" by the option value of the new scenario.

6. In the final step the new scenario has to be added to the body of the sub function GenerateInputSQLChecklist. Note that this works pretty much the same as in the previous step.

In fact all numerical values entered directly have to be equal to those entered in the previous step! If this is not the case SLAM may not find anything in the data base although it already saved a requested spectrum. Please note that one should apply the function StableNumber to real numbers in order to make them stable under the conversion from and to their FORTRAN form. If a number is not stable under these conversions, very small rounding errors occur, which spoil the detection of matching spectra in the data base.

Each numerical value follows an identification string in a list. This string can be built up from the position of the numerical value in a SLHA file as follows: Conventionally the first letter is a "I" which indicates that the value is entering the input value part of the SQL data base. Since white spaces are not allowed in SQL to be part of a column name, the separation to the following block name in capital letters is done by an underscore. After the block name the underscore separated key value follows as an integer. If it is a double key value, like used for matrix elements, there may be two integer numbers separated by one underscore. If there is no entry key at all, like 
it is the case for the output value of the mixing angle $\alpha$, which somehow got its own block, NONE replaces the usual integer number. One can check the adjustments by directly calling the function via:

SLAM'Private'GenerateInputSQLChecklist [Model -> "mgmsb"]

where "mgmsb" has to be replaced by the option value of the new scenario. The output should be just a list of pairs, where each pair is a list with an identification string as the first element and a numerical value as the second element.

Once the given steps have been completed, the new predefined scenario should work without any problems.

\section{Summary}

We presented and published the package SLAM, which provides a convenient interface for SLHA spectrum generators in Mathemati ca.

The package enables the user to obtain spectrum data from generators in a fully automatic way. Results of different spectrum generators can be compared without any effort and it allows the user to use his own notation in Mathematica. SLAM comes with a large number of built-in benchmark scenarios. Furthermore, it allows the user to freely define any desired scenario following the SLHA standard.

Moreover, it can store and recall all acquired data to and from a data base in order to avoid a recalculation of known spectra. Storing spectra in a data base allows the examination of parameter spaces by simply using powerful data base functionalities. A parallel use of SLAM is possible and helps to reduce the time needed for possible parameter scans and builds of data bases.

Besides the pure usage documentation including examples, we provided more details about the internal structure of the package which may help in case a modification of the program code is needed due to special user requirements.

\section{Acknowledgements}

We would like to thank M. Iskrzynski and A. Kurz for beta testing the application, and J. Hoff, A. Kurz and M. Steinhauser for reading the manuscript. Moreover we appreciate the fruitful time at the Institut für Theoretische Teilchenphysik at KIT, where the core routines of SLAM have been implemented and runtime benchmarks could be performed on the local PC cluster. This work has been supported in part by the EU Network LHCPHENOnet PITN-GA-2010-264564, and by DFG Sonderforschungsbereich Transregio 9, Computergestützte Theoretische Teilchenphysik.

\section{References}

[1] W. Porod, Comput. Phys. Commun. 153 (2003) 275. hep-ph/0301101

[2] B.C. Allanach, Comput. Phys. Commun. 143 (2002) 305. arXiv:hep-ph/0104145

[3] D. Chowdhury, R. Garani, S. KVempati, Comput. Phys. Commun. 184 (2013) 899. arXiv:1109.3551 [hep-ph].

[4] A. Djouadi, J.-L. Kneur, G. Moultaka, Comput. Phys. Commun. 176 (2007) 426. hep-ph/0211331.

[5] P.Z. Skands, B.C. Allanach, H. Baer, C. Balazs, G. Belanger, F. Boudjema, A. Djouadi, R. Godbole, et al., J. High Energy Phys. 0407 (2004) 036. hep-ph/0311123.

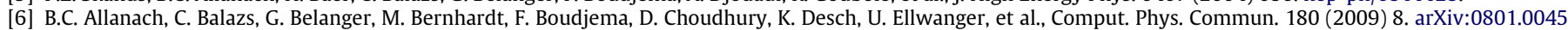
[hep-ph].

[7] http://home.fnal.gov/ skands/slha/.

[8] http://fthomas.github.com/slhaea/.

[9] http://www.svenkreiss.com/SLHAio.

[10] https://lhpc.hepforge.org/.

[11] G. Belanger, N.D. Christensen, A. Pukhov, A. Semenov, Comput. Phys. Commun. 182 (2011) 763. arXiv:1008.0181 [hep-ph]

[12] T. Hahn, hep-ph/0408283.

[13] T. Hahn, Comput. Phys. Commun. 180 (2009) 1681. hep-ph/0605049.

[14] http://www.insectnation.org/projects/pyslha.

[15] P. Kant, R.V. Harlander, L. Mihaila, M. Steinhauser, J. High Energy Phys. 1008 (2010) 104. arXiv:1005.5709 [hep-ph].

[16] A. Pak, M. Steinhauser, N. Zerf, arXiv:1208.1588 [hep-ph].

[17] A. Kurz, M. Steinhauser, N. Zerf, arXiv:1206.6675 [hep-ph].

[18] M.S. Carena, S. Heinemeyer, C.E.M. Wagner, G. Weiglein, Eur. Phys. J. C 26 (2003) 601. hep-ph/0202167.

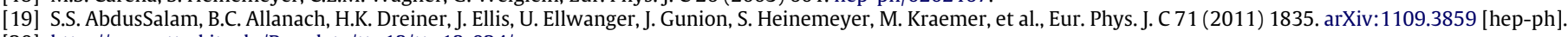

[20] http://www.ttp.kit.edu/Progdata/ttp13/ttp13-024/.

[21] M. Carena, S. Heinemeyer, O. Stål, C.E.M. Wagner, G. Weiglein, arXiv:1302.7033 [hep-ph].

[22] M.W. Cahill-Rowley, J.L. Hewett, A. Ismail, M.E. Peskin, T.G. Rizzo, arXiv:1305.2419 [hep-ph]. 